\title{
Distribution of COPD Comorbidities and Creation of Acute Exacerbation Risk Score: Results from SCICP
}

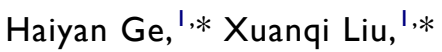 \\ Wenchao Gu, ${ }^{2}$ Xiumin Feng, ${ }^{3,4}$ \\ Fengying Zhang, ${ }^{5}$ \\ Fengfeng Han, ${ }^{6}$ Yechang Qian, ${ }^{7}$ \\ Xiaoyan Jin, ${ }^{8}$ Beilan Gao, \\ Li Yu, ${ }^{10}$ Hong Bao, ${ }^{11}$ \\ Min Zhou, ${ }^{12}$ Shengqing $\mathrm{Li},\left(\mathbb{D}^{13}\right.$ \\ Zhijun Jie, ${ }^{14}$ Jian Wang, ${ }^{15}$ \\ Zhihong Chen, ${ }^{16}$ \\ Jingqing Hang, ${ }^{5}$ Jingxi Zhang, ${ }^{3}$ \\ Huili Zhu'
}

'Department of Respiratory and Critical Care Medicine, Huadong Hospital, Fudan University, Shanghai, People's Republic of China; ${ }^{2}$ Department of Respiratory Medicine Pudong New District People's Hospital, Shanghai, People's Republic of China; ${ }^{3}$ Department of Respiratory and Criticl Care Medicine Changhai Hospital Aff Cricical Care Medicine, Changhai Hospital Affiliated to Navy Military Medical University, Shanghai, People's Republic of China; ${ }^{4}$ Department of Respiratory and Critical Care Medicine, Changji Branch of First Affiliated Hospital of Xinjiang Medical University, Xinjiang, People's Republic of China; ${ }^{5}$ Department of Respiratory Medicine Putuo District People's Hospital, Shanghai, People's Republic of China; ${ }^{6}$ Department of Respiratory Medicine Xinhu Xinhua Hospital, Shanghai liaotong University School of Medicine, Shanghai, People's Republic of China; 'Baosha District Hospital of Integrated Traditional Chinese and Western Medicine, Shanghai, People's Republic of China; ${ }^{8}$ Department of Respiratory Medicine, Tongren Hospital, Shanghai Jiaotong University School of Medicine, Shanghai, People's Republic of China; 'Department of Respiratory Medicine, Shanghai Pulmonary Hospital, Tongii University Shangin Shang Tongji University School of Medicine, Shanghai, People's Republic of China; "Department of Respiratory Medicine Shanghai Pudong Hospital, Fudan University Pudong Medical Center, Shanghai, People's Republic of China;

${ }^{12}$ Department of Respiratory and Critical Care Medicine, Ruijin Hospital, Shanghai Jiao Tong University School of Medicine, Shanghai, People's Republic of China;

${ }^{13}$ Department of Respiratory and Critical Care Medicine,

Huashan Hospital, Fudan University, Shanghai, People's

Republic of China; ${ }^{14}$ Department of Respiratory Medicine, Shanghai Fifth's Hospital, Fudan University Shanghai, People's Republic of China; ${ }^{15}$ Department of Respiratory Medicine, Shanghai Ninth's Hospital, Shanghai jiaotong University School of Medicine, Shanghai, People's Republic of China; ${ }^{16}$ Department of Respiratory and

Critical Care Medicine, Zhongshan Hospital, Fudan

University, Shanghai, People's Republic of China

*These authors contributed equally to this work

Correspondence: Huili Zhu

Email zhuhuili00I@I26.com

Jingxi Zhang

Email jingxizhang2000@I26.com
Background: Chronic obstructive pulmonary disease (COPD) often coexists with multiple comorbidities which may have a significant impact on acute exacerbations of patients. At present, what kind of comorbidities affects acute exacerbations and how comorbidities lead to poor prognosis are still controversial. The purpose of our study is to determine the impact of comorbidities on COPD exacerbation and establish an acute exacerbation risk assessment system related to comorbidities.

Methods: A total of 742 COPD patients participated in the Shanghai COPD Investigation on Comorbidity Program (SCICP, ChiCTR2000030911). Finally, the baseline information of 415 participants and one-year follow-up data were involved in the analysis. We collected hemogram indices, pulmonary function tests and acute exacerbation of COPD with regular medical follow-up. Q-type cluster analysis was used to determine the clusters of participants. Receiver operating characteristic (ROC) analysis was constructed to assess the ability of indicators in predicting acute exacerbations.

Results: Almost $65 \%$ of the population we investigated had at least one comorbidity. The distribution and incidence of comorbidities differed between exacerbation group and nonexacerbation group. Three comorbidity clusters were identified: (1) respiratory, metabolic, immune and psychologic disease (non-severe cases); (2) cardiovascular and neoplastic disease (severe cases); (3) less comorbidity. Different sub-phenotypes of COPD patients showed significant distinction in health status. Anxiety $(\mathrm{OR}=5.936, P=0.001)$, angina $(\mathrm{OR}=10.155, P=0.025)$ and hypertension $(\mathrm{OR}=3.142, P=0.001)$ were found to be independent risk factors of exacerbation in a year. The novel risk score containing BODEx and four diseases showed great prognostic value of COPD exacerbation in developing sample.

Conclusion: Our study detailed the major interaction between comorbidities and exacerbation in COPD. Noteworthily, a novel risk score using comprehensive index - BODEx - and comorbidity parameters can identify patients at high risk of acute exacerbation.

Keywords: chronic obstructive pulmonary disease, exacerbation, comorbidity, risk score

\section{Introduction}

Chronic obstructive pulmonary disease (COPD) is characterized by persistently incompletely reversible airflow limitation. It has always been a huge challenge to the world public health system and a heavy burden to individuals ${ }^{1,2}$. Until 2018 , COPD has ranked among the top three leading causes of death in China, and the incidence varied from $1.2 \% \sim 8.87 \%$ in different regions. ${ }^{3}$ COPD generally coexists with other chronic conditions ${ }^{4}$. To date, substantial evidence support that systematic inflammation and senescence (5) involved in the pathologic change of lungs in COPD. The systemic features of COPD include cardiovascular disease, metabolism 
disease, depression and so on. ${ }^{5}$ Multiple morbidities can interact with each other and exacerbate the problem. There's no doubt that the presence of multiple morbidities can change the internal microenvironment and homeostasis. ${ }^{6}$ However, the underlying common pathological pathway has not been totally figured out.

Acute exacerbation during COPD is the leading cause of worse outcome and disease progress which requires our great concern. ${ }^{7,8}$ Early recognition of patients with high exacerbation risk is considerably critical, so it is urgent to provide an effective tool to predict exacerbation. Until now, little attention has been paid to the complex relationship between comorbidity and exacerbation in individuals with COPD. On one hand, a variety of complicated score system of COPD like BODEx, COTE and ADO indices involved in diverse dimensions including age, BMI, lung function and so on, lacking comorbidities as one of the significant predictive factors. ${ }^{4}$ On the other hand, most researches focused on the relationship between score system and all-cause mortality of $\mathrm{COPD}^{9}$ rather than exacerbation event. In sum, further study should put emphasis on regarding comorbidity as one of the score items to predict COPD exacerbation.

In this prospective cohort study, we hope our study could increase the understanding of comorbidities' contribution to exacerbation in COPD. We developed a novel risk score based on comorbidity profile and conventional comprehensive indices (BODEx) in SCICP. To some extent, it may provide some assistance for assessing the risk of acute exacerbation of COPD.

\section{Method}

\section{Study Design and Patients}

We conducted Shanghai COPD Investigation on Comorbidity Program (SCICP), which is a 3-year observational cohort study, and registered at Chinese Clinical Trial Registry (ChiCTR2000030911). Written informed consent was available and the study was approved by the Ethics Committee of Huadong Hospital. Inclusion criteria: (1) The primary diagnosis is COPD which was complied with GOLD criteria (the forced expiratory volume in first second of forced vital capacity is $<70 \%$ after inhaling bronchodilators). ${ }^{10}$ (2) age over 40 years old. Exclusion criteria: (1) Have respiratory infection in recent 1 month. (2) Suffer from serious disease and unable to complete questionnaire.

\section{Procedures}

Information including demographic characteristics (gender, age, BMI, smoking history and exposure history) and clinical features (hemogram indices, lung function test, symptom score and comorbidities) was documented. Comorbidity related information concluded diseases of respiratory system (bronchiectasis, pulmonary embolism, asthma, allergic rhinitis and lung cancer), cardiovascular system (hypertension, heart failure, angina, arrhythmia) metabolism system (diabetes, osteoporosis, metabolism syndrome) nervous system (stroke, subarachnoid, dementia, hemiplegia) digestive system (peptic ulcer, liver disease, digestive tumor) and other diseases like peripheral vascular disease, connective tissue disease, leukemia, lymphoma, AIDS and anxiety. Exacerbation events of COPD patients were our primary endpoint event. In our study, the definition of COPD exacerbation has been strictly identified in operators' protocol according to GOLD2018. The occurrence of exacerbation means acute worsening of respiratory symptoms which results in additional therapy. Mild exacerbation was treated with short acting bronchodilators only. Moderate exacerbation should be treated with short acting bronchodilators plus antibiotics and/or oral corticosteroids. Severe exacerbation always requires hospitalization or emergency admission. Frequent exacerbation is defined as more than one exacerbation in a year. The participants in our study were under regular followup. The routine blood test, lung function test and symptom scores like mMRC score and CAT score were measured once a year. Apart from self-report, exacerbation event was acquired at 6-months interval via patients' diary, medication administration and electronic medication record.

\section{Statistical Analysis}

Continuous variables were presented as mean \pm standard deviation while categorical variables were expressed as percentages. In univariate analysis, data was evaluated by chi-square test, $t$-test or nonparametric test. Multivariate logistic regression model was constructed to determine the odds ratio (OR) and $95 \%$ confidence intervals $(95 \%)$ of independent risk in predicting exacerbation. We performed Receiver Operator Curve (ROC) and tabulated Area Under Curve (AUC) to measure the prognostic value of indexes in predicting exacerbation. Cluster analysis was performed to assign subjects into several groups with similar features without an 
anticipation. Q-type clustering analysis of subjects using comorbidity parameters was conducted by $\mathrm{K}$ means method and resulted in non-hierarchical cluster graph. We developing a novel risk score via nomogram. In all tests, two-sided $P$ value $<0.05$ was considered significant. While in multiple comparison, $P$-value $<0.05 / \mathrm{m}$ was considered significant after Bonferroni correction. All statistical analysis in our study was performed by STAT15 and graph was drawn by Adobe Illustrator 2020 .

\section{Results}

\section{The Distribution of Comorbidities in COPD Patients}

Among 742 participants enrolled in our study, 415 were eligible for analysis eventually. The detailed process is presented in Figure 1. All comorbidities of COPD patients are presented in Figure 2A, the frequencies of them ranged from $30.86 \%$ to $0.4 \%$. Hypertension, anxiety and allergic rhinitis ranked the most prevalent three comorbidities in order. The incidence rates of diseases including AIDS, leukemia and dementia were 0 in our study, which are not shown in Figure 2A. Almost $65 \%$ of the COPD patients we analyzed suffered from at least one comorbidity, and nearly $35 \%$ patients had more than two chronic conditions which was defined as patients with multiple diseases (Figure 2B). The co-existence of 26 comorbidities in COPD patients was presented in the heat map (Figure 2C). Among the whole subjects, we discovered that hypertension, as the most common COPD comorbidity, has a higher possibility of coexisting with asthma and allergic rhinitis. In COPD patients, the coexistence rate of anxiety and hypertension is also very high. Furthermore, we performed a subgroup analysis of COPD patients based on different characteristics (COPD course, age, gender, exacerbation and frequent exacerbation) and made a statistical comparison (Figure 2D). The results in Supplement 1A and B indicated that patients suffering from longer duration of COPD and male participants tended to have a higher incidence rate of comorbidity co-existence. No significant difference was observed between patients with

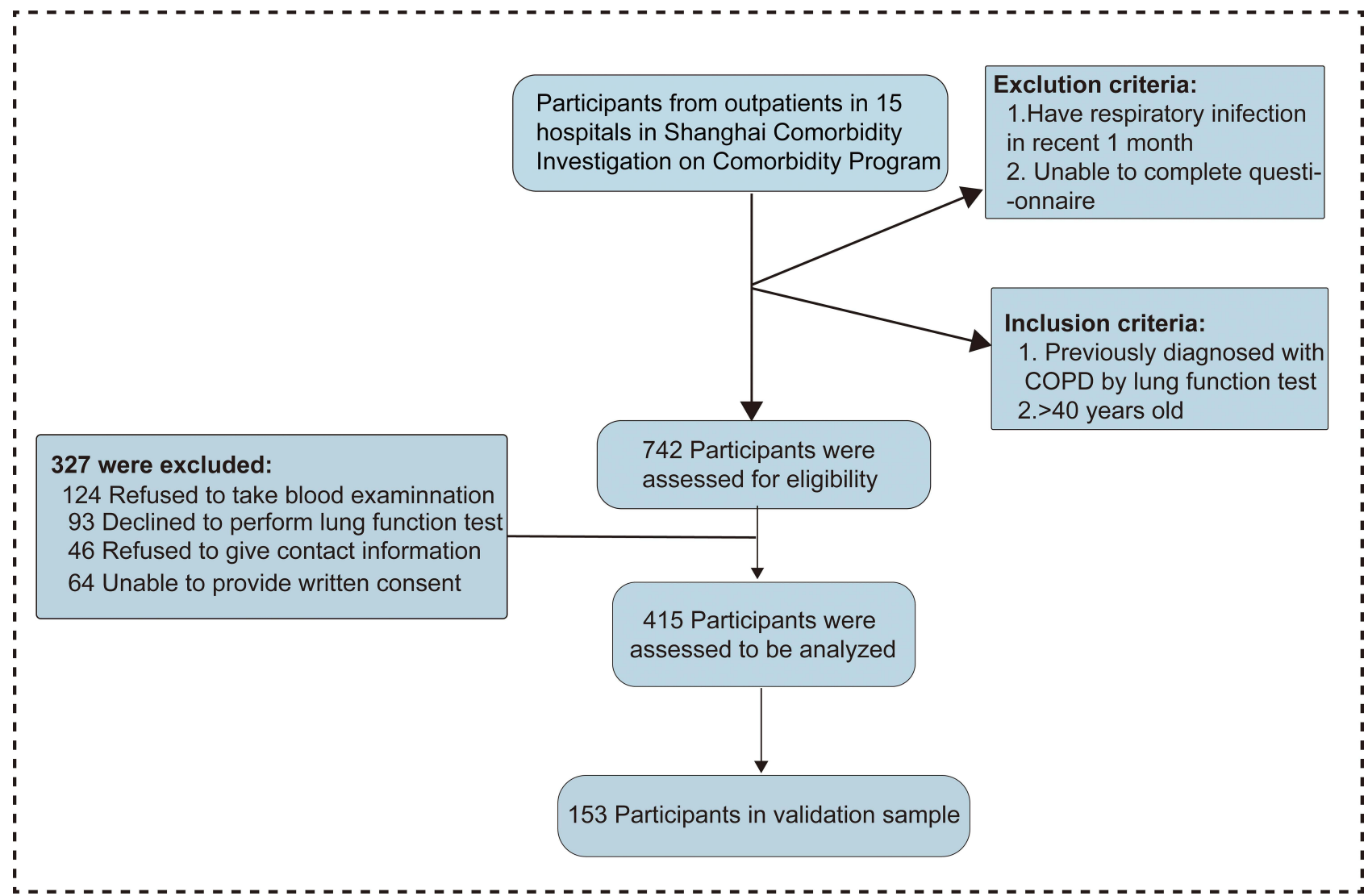

Figure I The process of the participants' enrollment. Flow diagram of participants in Shanghai COPD Investigation on Comorbidity Program. Abbreviation: COPD, chronic obstructive pulmonary disease. 
different ages (Supplement 1E). Exacerbations or frequent exacerbations mainly affected the distribution of comorbidity co-existence, although the incidence rate of comorbidity co-existence showed no significant differences between two groups (Supplement 1C and D). Exacerbation events were reported among patients with hypertension co-existed with asthma, anxiety and arrhythmia, while hypertension co-existing allergic rhinitis was found highest among patients without exacerbation.

\section{Comorbidity Clusters and Differences in Patients' Characteristics}

According to 26 kinds of comorbidity profile, three comorbidity clusters were identified among COPD patients (Figure 3A, Table 1): Cluster 1: Non-severe cases $(\mathrm{N}=99$, "Respiratory, Metabolic, Immune and Psychologic Disease"), Cluster 2: Severe cases ( $\mathrm{N}=16$, "Cardiovascular and Neoplastic Disease") and Cluster 3 ( $N=300$, "Less comorbidity"). Cluster 1 had the highest prevalence in asthma, allergic rhinitis, osteoporosis and connective tissue disease. Cluster 2 had a higher

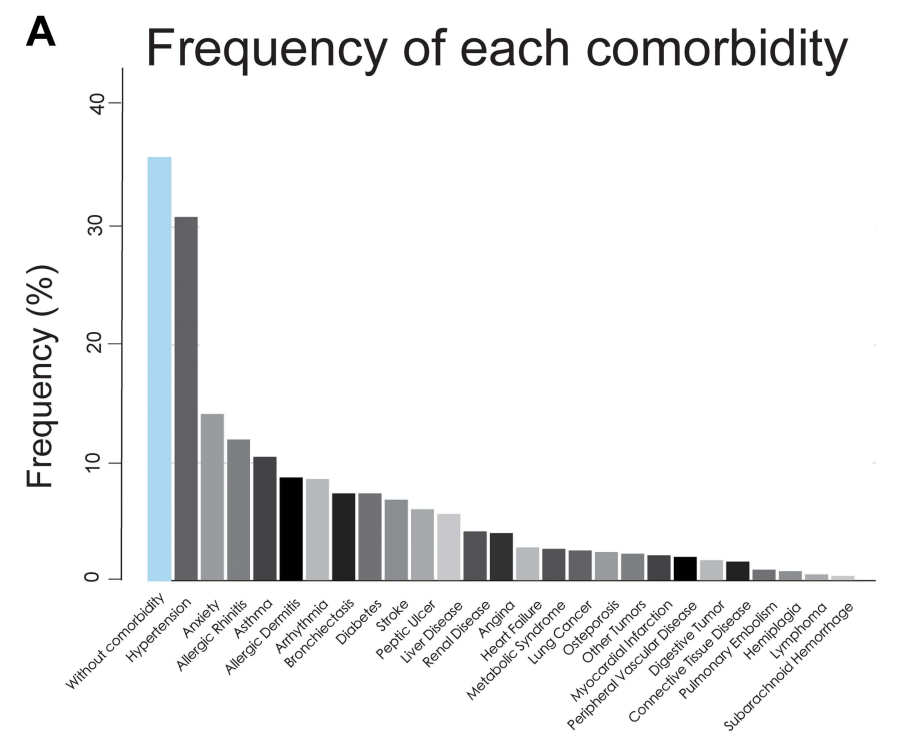

C

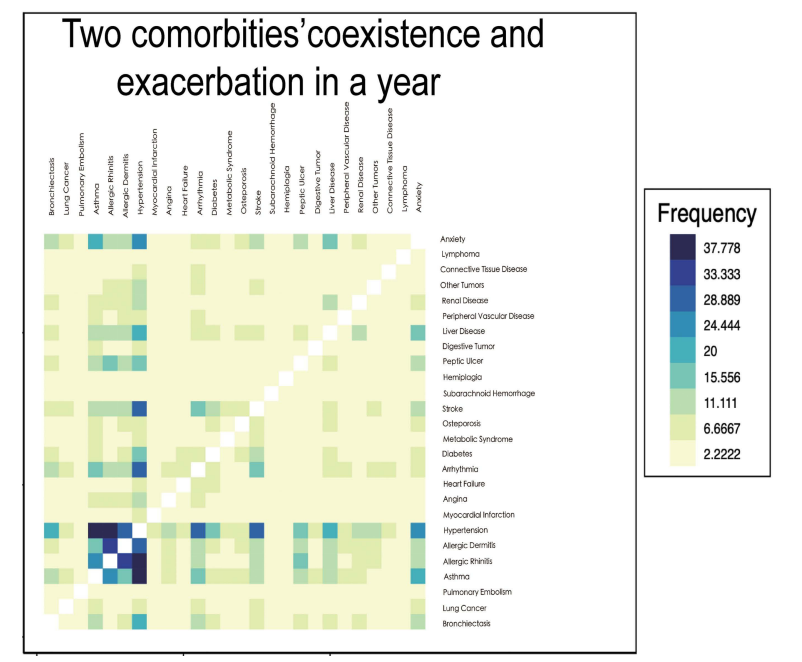

\section{B Number of comorbidity}

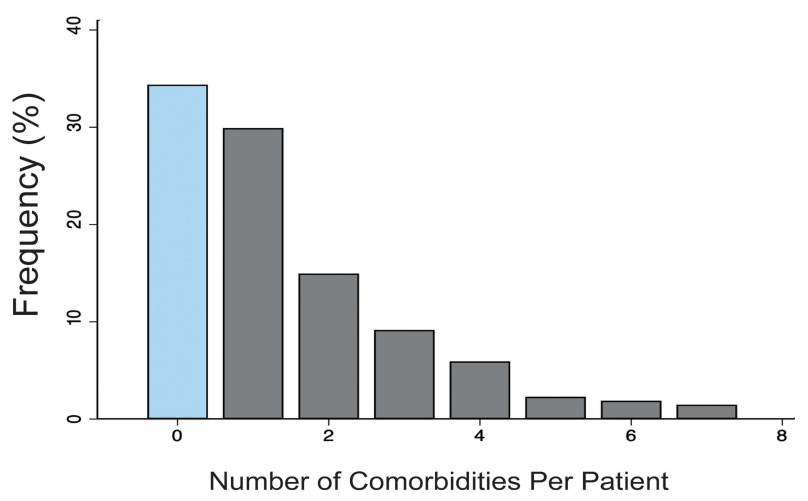

D

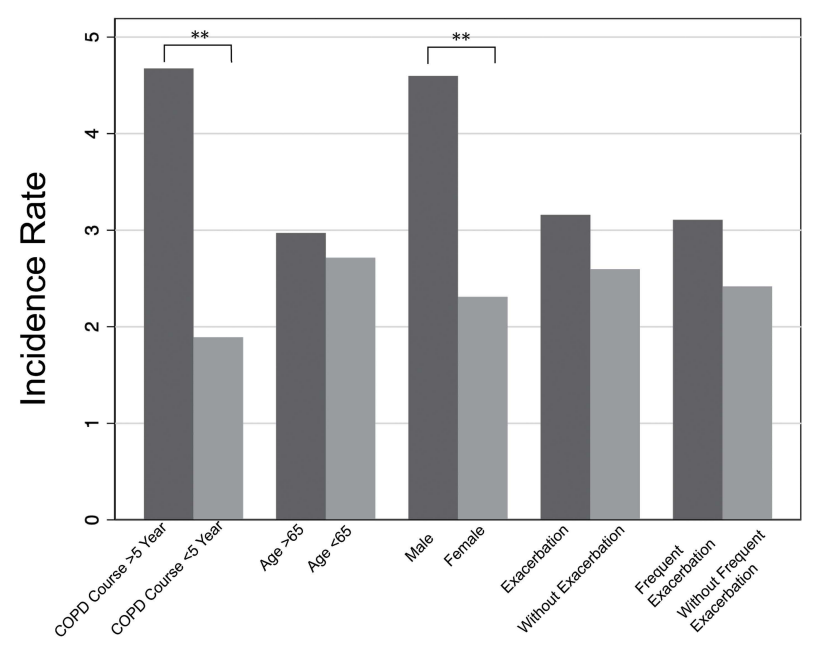

Figure 2 The distribution of comorbidity profile among COPD patients in Shanghai. (A) The frequency of each comorbidity in the training cohort. (B) The number of comorbidities per patient suffered in the training cohort. (C) The heat map for the two comorbidities' coexistence in the training cohort depicted the correlation between any two kinds of comorbidity. The row and column showed 26 kinds of comorbidities identified among COPD patients. For interpretation, the color of blue represented the highest value while the color of yellow represented the lowest value. (D) The incidence rates of comorbidities' coexistence were compared between different groups in training cohort $(* * P<0.05)$. 
A
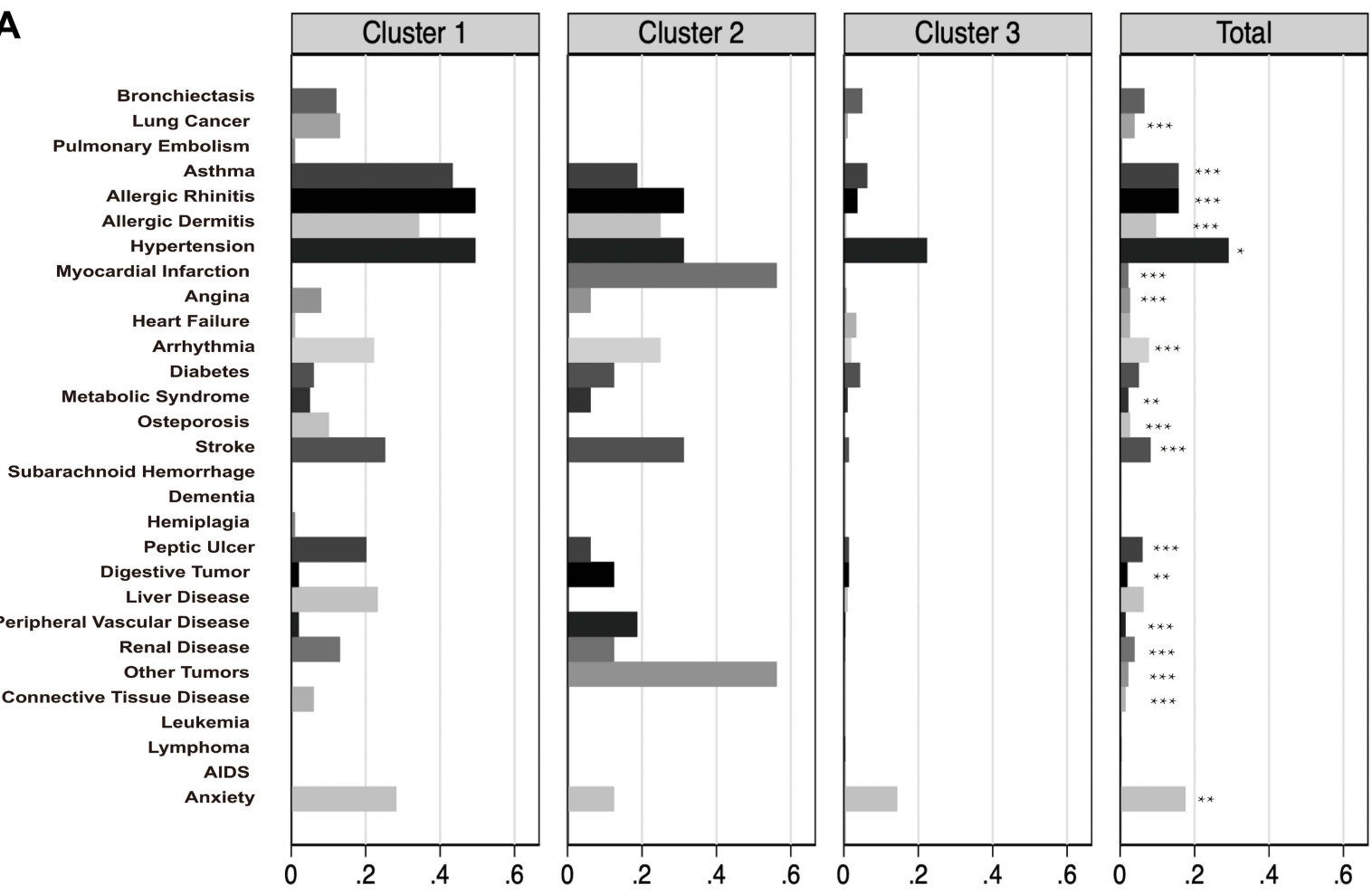

B

Peptic Ulcer
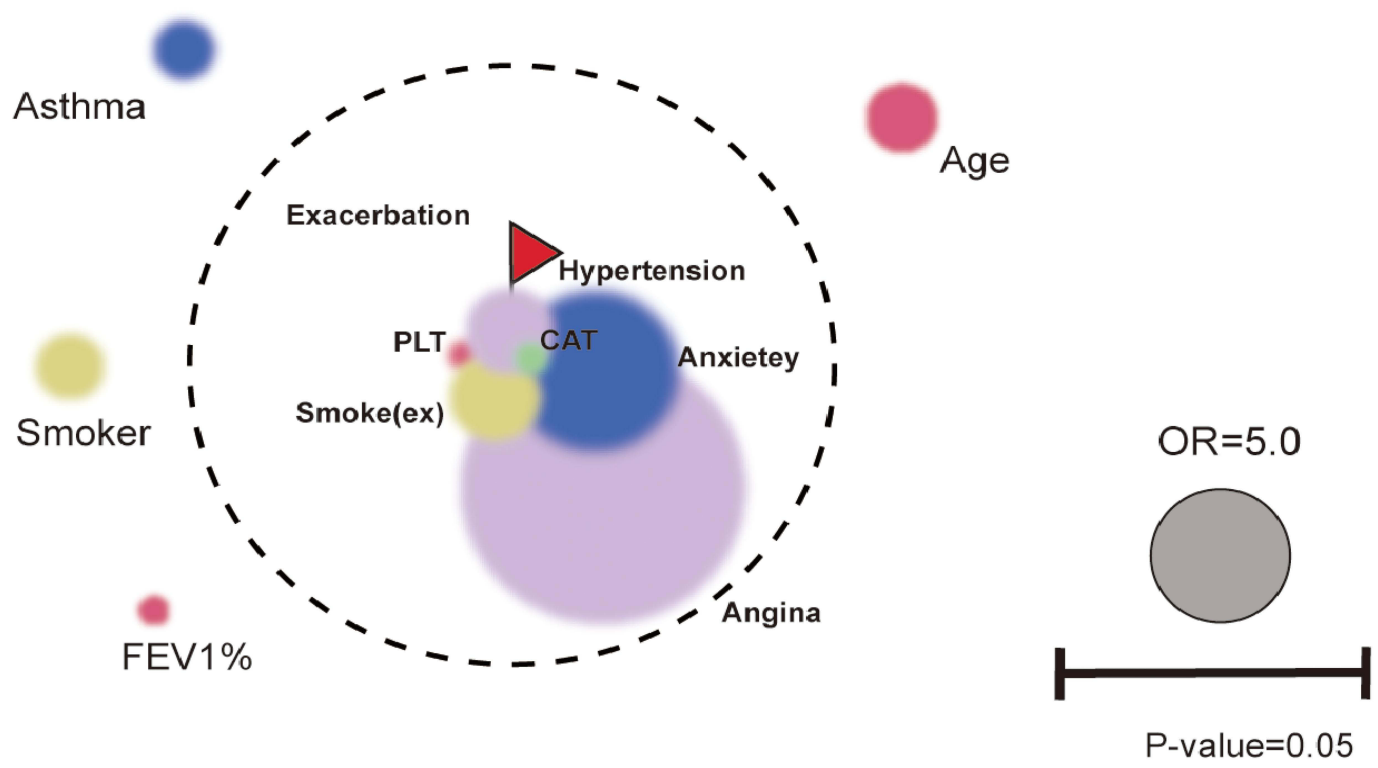

Figure 3 Development of new score and application in different clusters of COPD patients. (A) The incidence rate of different comorbidities is compared among three different clusters. The cluster analysis is based on the 26 kinds of comorbidities. Cluster I(Respiratory disease, Metabolic Disease, immune disease), Cluster 2 (Cardiovascular disease, Neoplastic disease) and Cluster 3(less comorbidities). ( $* P<0.1, * * P<0.05, * * * P<0.00 \mathrm{I})$. (B) Multivariate logistic regress analysis identified the independent risk factors for acute exacerbation of COPD in a year. The area of the circle positively relates to odds ratio (OR) in multivariate model. The proximity to the center (i.e exacerbation) represented the minor P-value. Bubble colors expressed different types of risk events (comorbidity profile, baseline features and clinical characteristics). 
Table I Comorbidity Related Information Among Patients in 3 Clusters

\begin{tabular}{|c|c|c|c|c|c|c|c|}
\hline & Total & Cluster I & Cluster 2 & Cluster 3 & $\mathbf{P}^{\mathbf{a}}$ & $\mathbf{P}^{\mathbf{b}}$ & $\mathbf{P}^{\mathbf{c}}$ \\
\hline Number of Subject & 415 & 99 & 16 & 300 & - & - & - \\
\hline Number of Comorbidity & 2.7 & 3.77 & 3.63 & 0.71 & 1.000 & $<0.001$ & $<0.001$ \\
\hline Bronchiectasis & $27(6.5 I)$ & $12(12.12)$ & $0(0)$ & $15(5.00)$ & 0.202 & 0.038 & 1.000 \\
\hline Lung Cancer & $16(3.86)$ & $13(13.13)$ & $0(0)$ & $3(1.00)$ & 0.027 & $<0.001$ & 1.000 \\
\hline Pulmonary Embolism & $2(0.48)$ & $\mathrm{I}(\mathrm{I} .0 \mathrm{I})$ & $0(0)$ & $\mathrm{I}(\mathrm{I} .33)$ & 1.000 & 1.000 & 1.000 \\
\hline Asthma & $65(15.66)$ & $43(43.43)$ & $3(18.75)$ & $19(6.33)$ & 0.017 & $<0.001$ & 0.426 \\
\hline Allergic Rhinitis & $65(15.66)$ & $49(49.19)$ & $5(31.25)$ & $\mathrm{II}(3.67)$ & 0.084 & $<0.001$ & 0.002 \\
\hline Allergic Dermatitis & $40(9.64)$ & $34(34.34)$ & $4(25.00)$ & $2(0.67)$ & 0.536 & $<0.001$ & 0.001 \\
\hline Hypertension & $121(29.16)$ & $49(49.49)$ & $5(31.25)$ & $67(22.33)$ & 0.377 & $<0.001$ & 1.000 \\
\hline Myocardial Infarction & $9(2.17)$ & $0(0)$ & $9(56.25)$ & $0(0)$ & $<0.001$ & 1.000 & $<0.001$ \\
\hline Angina & $\mathrm{II}(2.65)$ & $8(8.08)$ & $I(6.25)$ & $2(0.67)$ & 1.000 & $<0.001$ & 0.507 \\
\hline Heart Failure & $\mathrm{II}(2.65)$ & $\mathrm{I}(\mathrm{I} .0 \mathrm{I})$ & $0(0)$ & $10(3.33)$ & 1.000 & 0.640 & 1.000 \\
\hline Arrhythmia & $32(7.7 I)$ & $22(22.20)$ & $4(25.00)$ & $6(2.00)$ & 1.000 & $<0.001$ & 0.001 \\
\hline Diabetes & $2 \mathrm{I}(5.06)$ & $6(6.06)$ & $2(12.5)$ & $13(4.33)$ & 0.830 & 1.000 & 0.443 \\
\hline Metabolism Syndrome & $9(2.17)$ & $5(5.05)$ & $\mathrm{I}(6.25)$ & $3(1.00)$ & 1.000 & 0.049 & 0.476 \\
\hline Osteoporosis & $\mathrm{II}(2.65)$ & $10(10.10)$ & $0(0)$ & $I(0.33)$ & 0.049 & $<0.001$ & 1.000 \\
\hline Stroke & $34(8.19)$ & $25(25.25)$ & $5(3 \mid .25)$ & $4(1.33)$ & 1.000 & $<0.001$ & $<0.001$ \\
\hline Sub-arachonoid & - & - & - & - & - & - & - \\
\hline Hemiplegia & $\mathrm{I}(0.24)$ & $\mathrm{I}(1.0 \mathrm{I})$ & $0(0)$ & $0(0)$ & 1.000 & 0.228 & 1.000 \\
\hline Peptic Ulcer & $25(6.02)$ & $20(20.20)$ & $\mathrm{I}(6.25)$ & $4(1.33)$ & 0.065 & $<0.001$ & 1.000 \\
\hline Digestive Tumor & $8(1.93)$ & $2(2.02)$ & $2(12.5)$ & $4(1.33)$ & 0.014 & 1.000 & 0.005 \\
\hline Liver Disease & $26(6.27)$ & $23(23.23)$ & $0(0)$ & $3(1.00)$ & $<0.001$ & $<0.001$ & 1.000 \\
\hline Peripheral vascular Disease & $6(1.45)$ & $2(2.02)$ & $3(18.75)$ & $\mathrm{I}(0.33)$ & $<0.001$ & 0.612 & $<0.001$ \\
\hline Renal Disease & $16(3.86)$ & $13(13.13)$ & $2(12.5)$ & $\mathrm{I}(0.33)$ & 1.000 & $<0.001$ & 0.032 \\
\hline Connective Tissue Disease & $6(1.45)$ & $6(6.06)$ & $0(0)$ & $0(0)$ & 0.165 & $<0.001$ & 1.000 \\
\hline Lymphoma & $I(0.24)$ & $0(0)$ & $0(0)$ & $\mathrm{I}(0.33)$ & 1.000 & 1.000 & 1.000 \\
\hline Anxiety & $73(17.59)$ & $28(28.28)$ & $2(12.5)$ & $43(\mid 4.33)$ & 0.364 & 0.005 & 1.000 \\
\hline Other solid tumors & $9(2.17)$ & $0(0)$ & $9(56.25)$ & $0(0)$ & $<0.001$ & 1.000 & $<0.001$ \\
\hline
\end{tabular}

Notes: ${ }^{a}$ Nonparametric test, $\mathrm{P}^{\mathrm{a}}$ indicated the significance between Cluster I and Cluster $2 .{ }^{\mathrm{b}}$ Nonparametric test, $\mathrm{P}^{\mathrm{b}}$ indicated the significance between Cluster I and Cluster 3. ${ }^{c}$ Nonparametric test, $\mathrm{P}^{\mathrm{c}}$ indicated the significance between Cluster 2 and Cluster 3.

prevalence in myocardial infarction, arrhythmia and neoplastic diseases, especially gastrointestinal tumors. On average, patients in Cluster 3 suffered from less diseases than patients in other clusters. Some clinical parameters were different from these three clusters. Although patients in Cluster 3 had the fewest number of comorbidities, they had worse pulmonary function (FEV1\%) and higher degree of airflow limitation. The 
results in our study demonstrated that population in cluster 2 gained more in comorbidity specified scores (including CCI index, age adjusted CCI index and COTE index). Cluster 1 (Respiratory, Metabolic, Immune and Psychologic Disease) appeared to elevated platelet and hemoglobin count which indicated better health-related condition compared with Cluster 2. It was implicated that longer biofuel exposure history in Cluster 1 was closely related to the respiratory diseases (Table 2).

Table 2 Clinical Characteristics Among Patients in 3 Clusters

\begin{tabular}{|c|c|c|c|c|c|c|}
\hline & Cluster I & Cluster 2 & Cluster 3 & $\mathbf{P}^{\mathbf{a}}$ & $\mathbf{P}^{\mathbf{b}}$ & $\mathbf{P}^{c}$ \\
\hline Number of Subject & 99 & 16 & 300 & - & - & - \\
\hline Gender (Male/Female) & $87 / 12$ & $14 / 2$ & $263 / 37$ & 1.000 & 1.000 & 1.000 \\
\hline Age & $68.44 \pm 8.25$ & $76.44 \pm 9.94$ & $71.06 \pm 9.55$ & 0.004 & 0.046 & 0.073 \\
\hline BMI & $23.8 I \pm 3.99$ & $24.88 \pm 4.97$ & $23.82 \pm 4.32$ & 1.000 & 1.000 & 1.000 \\
\hline $\begin{array}{l}\text { Smoke Status (Never/ex/ } \\
\text { smoker) }\end{array}$ & $27 / 44 / 28$ & $5 / 8 / 3$ & $85 / 165 / 50$ & 1.000 & 0.334 & 1.000 \\
\hline COPD Course (Years) & $5.48 \pm 7.67$ & $9.29 \pm 11.06$ & $8.01 \pm 7.38$ & 0.193 & 0.013 & 1.000 \\
\hline GOLD Stage (I/II/III//IV) & $\begin{array}{l}12 / 39 / 39 / 9(12 \% / 39 \% / \\
39 \% / 9 \%))\end{array}$ & $\begin{array}{l}5 / 5 / 4 / 2(31 \% / 3 \mid \% / 25 \% / \\
13 \%)\end{array}$ & $\begin{array}{l}|7 / 58 /| 78 / 47(6 \% / 19 \% / 59 \% / \\
16 \%)\end{array}$ & 0.610 & $<0.001$ & 0.003 \\
\hline Chemical Exposure & $\mathrm{II}(\mathrm{II.II)}$ & $2(12.50)$ & $17(5.67)$ & 1.000 & 0.210 & 0.911 \\
\hline Biofuel Exposure & $17(17.17)$ & $2(12.50)$ & II(3.67) & 1.000 & $<0.001$ & 0.524 \\
\hline Times of exacerbation & $1.25 \pm 1.40$ & $1.38 \pm 1.96$ & $1.14 \pm 2.12$ & 1.000 & 1.000 & 1.000 \\
\hline mMRC scores $(0 / 1 / 2 / 3 / 4)$ & $13 / 29 / 35 / \mid 8 / 4$ & $0 / 7 / 3 / 6 / 0$ & $35 / 132 / 77 / 50 / 6$ & 1.000 & 0.387 & 0.332 \\
\hline CAT scores & $15.09 \pm 7.59$ & $13.19 \pm 5.92$ & $14.42 \pm 7.42$ & 1.000 & 1.000 & 1.000 \\
\hline Neutrophil(10^9/L) & $5.16 \pm 2.52$ & $4.23 \pm 1.44$ & $5.68 \pm 2.86$ & 0.618 & 0.306 & 0.117 \\
\hline Eosnophils(10^9/L) & $0.18 \pm 0.18$ & $0.22 \pm 0.13$ & $0.20 \pm 0.19$ & 1.000 & 1.000 & 1.000 \\
\hline Platelet(10^9/L) & $206.79 \pm 60.20$ & $203.25 \pm 50.06$ & $183.20 \pm 77.68$ & 1.000 & 0.017 & 0.861 \\
\hline Hemoglobin(g/L) & $|40.7| \pm \mid 3.44$ & $137.69 \pm 15.64$ & $133.20 \pm 23.72$ & 1.000 & 0.008 & 1.000 \\
\hline FEVI\% & $53.99 \pm 21.21$ & $62.33 \pm 25.98$ & $43.78 \pm 19.92$ & 0.396 & $<0.001$ & 0.001 \\
\hline $\mathrm{FEVI} / \mathrm{FVC} \%$ & $57.59 \pm 11.78$ & $58.91 \pm 13.37$ & $54.00 \pm 12.74$ & 1.000 & 0.042 & 0.382 \\
\hline $\mathrm{CCl}$ Index & $1.98 \pm 1.91$ & $2.88 \pm 1.45$ & $0.3 \pm 0.65$ & 0.009 & $<0.001$ & $<0.001$ \\
\hline Age adjusted $\mathrm{CCl}$ Index & $4.23 \pm 2.03$ & $5.63 \pm 1.45$ & $2.73 \pm 0.88$ & $<0.001$ & $<0.001$ & $<0.001$ \\
\hline COTE Index & $2.93 \pm 3.69$ & $5.00 \pm 3.97$ & $1.09 \pm 3.33$ & 0.018 & $<0.001$ & $<0.001$ \\
\hline ADO Index & $4.14 \pm 1.64$ & $4.75 \pm 1.81$ & $4.66 \pm 1.62$ & 0.502 & 0.020 & 1.000 \\
\hline DOSE Index & $2.53 \pm 1.56$ & $2.50 \pm 1.75$ & $2.59 \pm 1.61$ & 1.000 & 1.000 & 1.000 \\
\hline BODEx Index & $2.90 \pm 1.99$ & $2.50 \pm 2.03$ & $3.26 \pm 1.73$ & 1.000 & 0.247 & 0.300 \\
\hline CODEx Index & $3.59 \pm 1.97$ & $3.31 \pm 2.18$ & $4.02 \pm 1.68$ & 1.000 & 0.115 & 0.360 \\
\hline
\end{tabular}

Notes: ${ }^{\mathrm{a}}$ Nonparametric test, $\mathrm{P}^{\mathrm{a}}$ indicated the significance between Cluster I and Cluster 2. ${ }^{\mathrm{b}}$ Nonparametric test, $\mathrm{P}^{\mathrm{b}}$ indicated the significance between Cluster I and Cluster 3. ${ }^{c}$ Nonparametric test, $\mathrm{P}^{c}$ indicated the significance between Cluster 2 and Cluster 3. 
The Relationship Between Comorbidities, Clinical Features, Healthy Features and Exacerbations

Three models of multi-variate logistic regression demonstrated that several kinds of diseases were in strong relationship with COPD exacerbation. We presented the variables of $p$-value less than 0.1 in Table 3 , and the complete results of logistic regression model was in Supplement 2. In the crude regression model, anxiety was the most significant risk factor $(\mathrm{OR}=7.415, P<0.001)$ (Table 3). After adjusting for potential confounding factors, hypertension, angina and anxiety were independent risk factors of exacerbation in COPD patients. However, there were relatively few COPD patients with angina in our study. The data was considerably weak and should be used with precaution. Additionally, we found that patients with allergic rhinitis appeared to have less exacerbation events. Apart from the comorbidity-related information, the elevated platelet count and CAT score were also independent risk factors. In accordance with the results in model 3, we presented it in the bubble plot (Figure 3B).

\section{Development of Scoring System to Predict Risk of Acute Exacerbation in COPD Patients}

To better predict the acute exacerbation of COPD, we compared the predictive ability of comprehensive indexes (Figure 4A). These comprehensive indexes could be divided into two parts: comorbidity-specific index (CCI, age adjusted CCI, COTE, CODEx) and comorbiditynonspecific index (ADO, BODEx). The comorbidityspecific index referred to the index consisted of comorbidity information while the nonspecific index was not. The results demonstrated that the predictive ability of CODEx $(\mathrm{AUC}=0.672)$ and BODEx $(\mathrm{AUC}=0.6685)$ exceeded far from other indexes (Figure 4A). We further combined the index BODEx with the following four diseases (hypertension, allergic rhinitis, angina and anxiety) which were closely related to exacerbations. Based on these four comorbidities and BODEx, we established a novel score system via nomogram plot. Because of the fewer subjects in Cluster 2 than in Cluster 1 and Cluster 3, there was few endpoints (exacerbation) in Cluster 2. Furthermore, we also identified a better prognostic value of the new score in Cluster $1(\mathrm{AUC}=0.8000)$ compared with Cluster 3 (AUC $=0.7656) \quad$ (Figure $4 \mathrm{C})$. From the results in
Figure 4B, we can see that angina was the most contributing factor. In detail, when the patients suffered from angina, the score in exacerbation system will increase 6.7 points. Additionally, developing anxiety and hypertension would respectively add 5.4 and 2.7 points in exacerbation system. The predictive efficacy was expressed in Figure 4A, the AUC of BODEx was significantly elevated after combining with other 4 diseases. For every 0.8 units increase in BODEx, the score in exacerbation system would increase by 1 point (Figure 4B).

\section{The Distribution of the Score and Its Prediction in Validation Sample}

As shown in Figure 5A, the novel score was in a nearly normal distribution. According to the results in ROC graph, we determined that the cutoff value of the new score was 3.6. The fraction of high score was larger among patients in GOLD IV than in GOLD I (Figure 5B). The novel score we developed also showed superiority in discerning patients with worse symptoms (mMRC score) (Figure 5C). Furthermore, we explored the correlation between the novel score and lung function including $\mathrm{FEV} 1 \%$ and $\mathrm{FEV} 1 / \mathrm{FVC} \%$, and the score was in negative relationship with them and showed significant difference among three clusters (Figure 5D and E). As we mentioned before, cluster 2 was characterized by severe illness. With the increase in score, lung function in cluster 2 dropped most compared with other clusters. In the internal validation sample, a total of 152 participants' exacerbation event was tracked and documented. We found that the score system still behaved well in the validation cohort $(\mathrm{AUC}=0.7195)$ (Table 4). Furthermore, we explored the predictive ability of scoring system in different degrees of exacerbation, respectively. Finally, we discovered that the scoring system containing BODEx, angina, hypertension, anxiety and allergic Rhinitis was more appropriate in predicting mild exacerbation $(\mathrm{AUC}=0.7361)$ as shown in Table 4.

\section{Discussion}

We established a three-year prospective cohort (SCICP), and demonstrated that hypertension, angina and anxiety were independent risk factor of acute exacerbation among COPD patients in Shanghai. Moreover, we developed a novel risk score from the cohort study to predict acute exacerbation of COPD patients. The risk score incorporates four comorbidities and BODEx index. The new score 
Table 3 Logistic Regression Between Exacerbation in One Year and Comorbidity, Clinical Characteristics

\begin{tabular}{|c|c|c|c|c|c|c|c|c|c|}
\hline \multirow[t]{2}{*}{ Model } & \multicolumn{3}{|c|}{ Crude Model } & \multicolumn{3}{|c|}{ +Clinical Features } & \multicolumn{3}{|c|}{ +Healthy Features } \\
\hline & OR & $95 \% \mathrm{Cl}$ & $P$-value & OR & $95 \% \mathrm{Cl}$ & $P$-value & OR & $95 \% \mathrm{Cl}$ & $P$-value \\
\hline Asthma & 1.821 & $0.913-3.630$ & 0.089 & 1.964 & $0.933-4.133$ & 0.075 & 2.102 & $0.922-4.795$ & 0.077 \\
\hline Allergic Rhinitis & 0.491 & $0.244-0.987$ & 0.046 & 0.384 & $0.186-0.794$ & 0.010 & 0.268 & $0.114-0.628$ & 0.002 \\
\hline Hypertension & 1.969 & I. $192-3.250$ & 0.008 & 2.695 & $1.546-4.697$ & $<0.001$ & 3.142 & $1.581-6.244$ & 0.001 \\
\hline Angina & 7.436 & $0.863-64.034$ & 0.068 & 9.487 & $0.998-90.146$ & 0.050 & 10.155 & $|.977-| 05.54 \mid$ & 0.025 \\
\hline Peptic Ulcer & 2.424 & $0.84 I-6.987$ & 0.101 & 2.901 & $0.970-8.765$ & 0.059 & 2.819 & $0.823-9.664$ & 0.099 \\
\hline Peripheral vascular Disease & 0.117 & $0.010-1.340$ & 0.085 & 0.105 & $0.012-0.952$ & 0.045 & 0.138 & $0.011-1.697$ & 0.122 \\
\hline Anxiety & 7.415 & $3.546-15.507$ & $<0.001$ & 13.528 & $5.854-31.260$ & $<0.001$ & 5.936 & $2.156-16.342$ & 0.001 \\
\hline Age & - & - & - & 1.935 & $0.224-1.194$ & 0.122 & 2.515 & $0.143-1.103$ & 0.076 \\
\hline BMI & - & - & - & 0.952 & $0.899-1.008$ & 0.093 & 0.988 & $0.916-1.066$ & 0.765 \\
\hline Smoker & - & - & - & 3.747 & $1.983-7.079$ & $<0.001$ & 3.209 & $|.464-7.03|$ & 0.004 \\
\hline Ex-smoker & - & - & - & 4.813 & $2.189-10.58 \mid$ & $<0.001$ & 2.400 & $0.926-6.221$ & 0.072 \\
\hline Expose Biofuel & - & - & - & 0.381 & $0.128-1.130$ & 0.487 & 0.233 & $0.072-0.758$ & 0.015 \\
\hline CAT scores & - & - & - & - & - & - & 1.162 & $1.102-1.226$ & $<0.001$ \\
\hline FEVI\% & - & - & - & - & - & - & 1.036 & $0.997-1.077$ & 0.071 \\
\hline Platelet & - & - & - & - & - & - & 1.006 & $1.002-1.011$ & 0.008 \\
\hline
\end{tabular}

shows better predictive capacity than other existing score system. Besides, we found that the risk score is more appropriate in population co-existing with Respiratory, Metabolic, Immune and Psychologic Disease (Cluster 1: non-severe cases). When being internally validated, the novel risk score could discriminate patients with or without exacerbation well according to the data from validation cohort. Although there have been several cohort studies validating the efficacy of index in COPD, for example, Almagro et al focused on CODEx index and mortality of COPD patients, ${ }^{11}$ and Divo et al proposed that the COTE index helped assess the mortality risk. ${ }^{12}$ Another study explored 6 basic parameters and medication patterns as indicator to predict mortality and worse SGRQ score. ${ }^{13}$ The specificity of our study is that the new score derived from comorbidity profile and important clinical features. More importantly, we focused on the exacerbation events rather than mortality in COPD patients. In our study, we hope to display the data of comorbidities in Shanghai and provide some assistance for assessing the risk of acute exacerbation of COPD.

COPD has been the third cause of death in the world. Moderate-to-severe COPD tends to call for a considerable economic burden for providers. ${ }^{14}$ The problem may be aggravated by exacerbation event. Comorbidity profile in COPD precisely makes them susceptible to frequent exacerbation. ${ }^{15}$ Previous research found that comorbidities seemed to develop at an earlier age and there was a higher prevalence in population with COPD when compared with general population. ${ }^{16}$ In elder patients with COPD, inhaler techniques, adherence, respiratory comorbidities and even psychologic disorder (cognitive impairment) should be regarded as predictive tools for clinical risk ${ }^{17}$. The results in our study described part of comorbidities and emphasized the importance of cardiovascular event in COPD patients.

It is first suggested in GOLD 2021 that there is an increased risk of ischemic heart disease during COPD exacerbation and even within 90 days. ${ }^{18}$ Consistently, our 
A
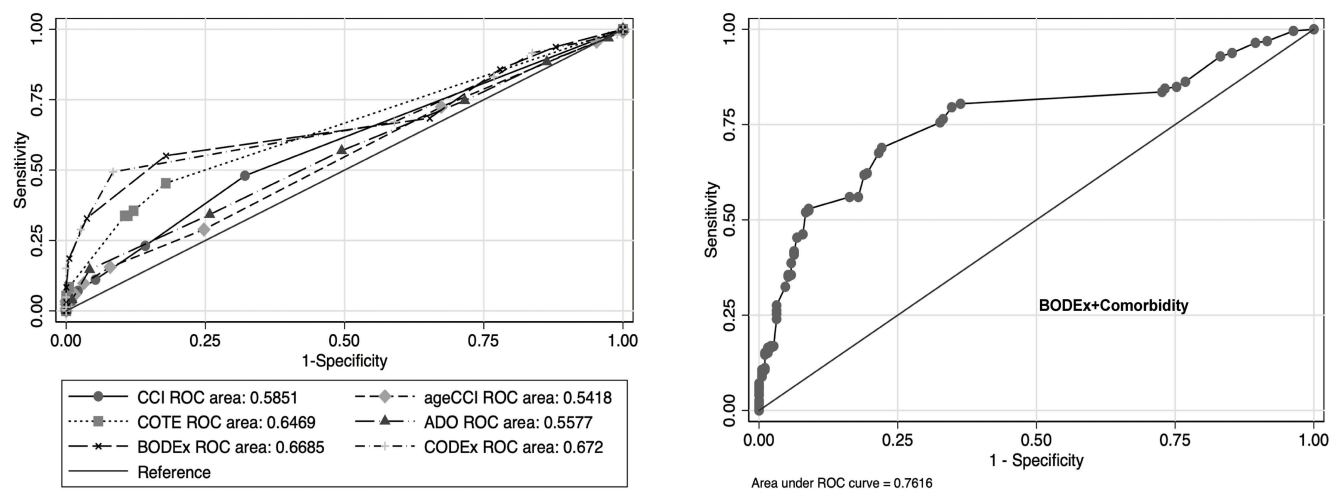

B
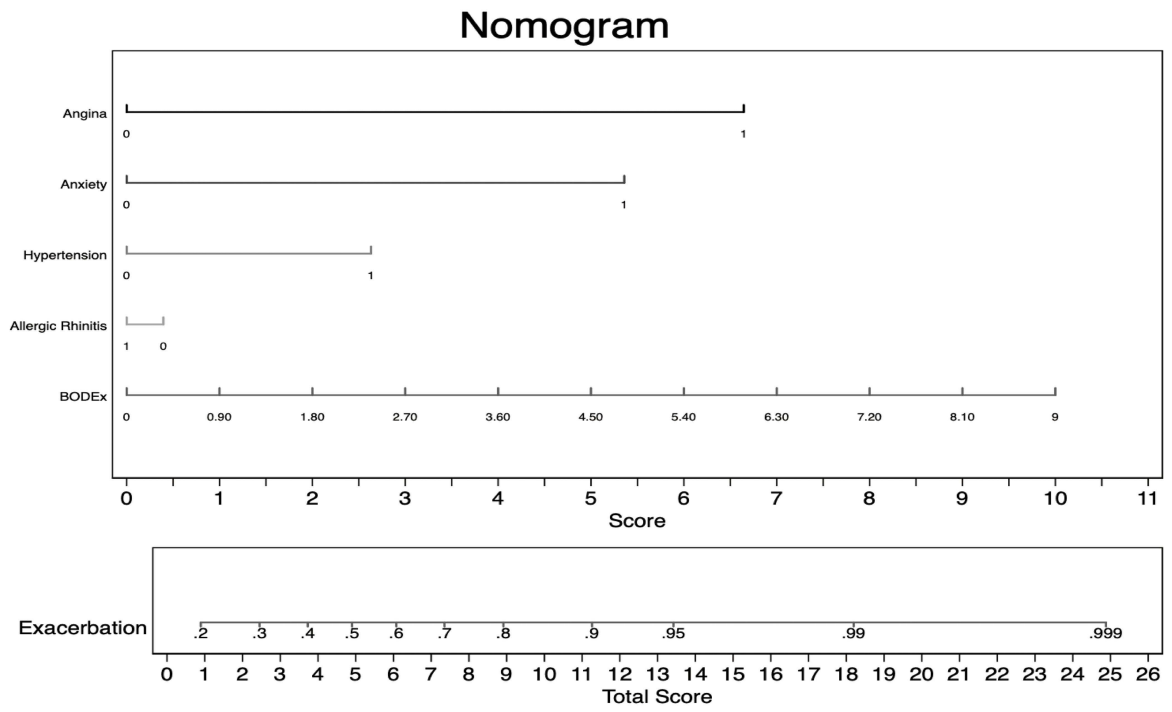

C

The prediction of new score in cluster 1
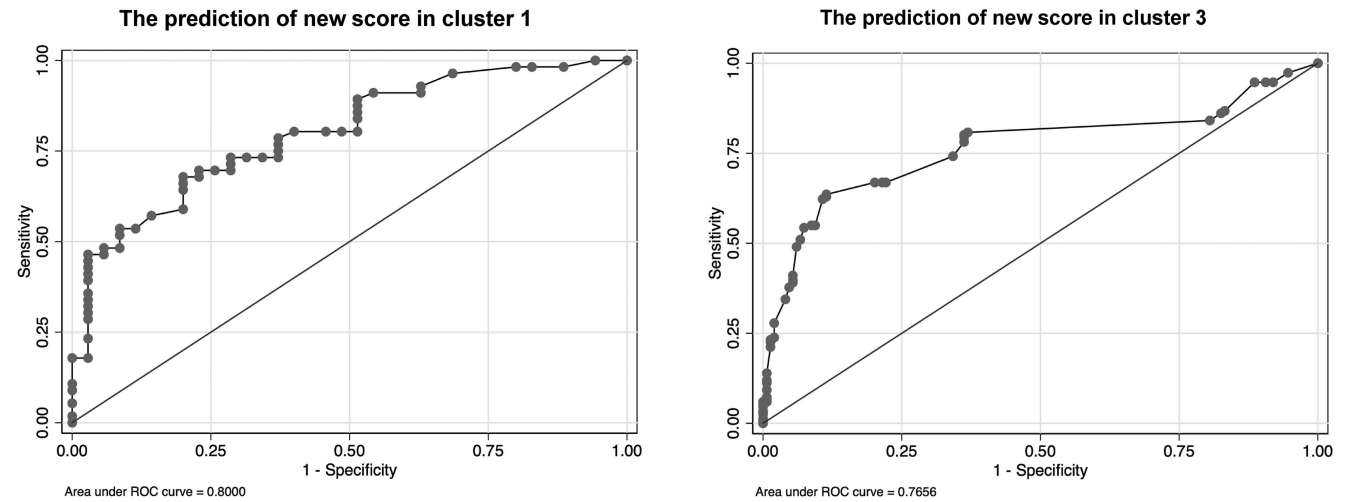

Figure 4 The discrimination and validation of new score in training cohort. (A) ROC analysis for each indice (CCI, age adjusted CCI, ADO, COTE, CODEx and BODEx) to predict acute exacerbation of COPD in a year and new score (combination of BODEx and comorbidity profile). $\mathrm{CCl}$ index concludes 13 kinds of comorbidities. Age adjusted $\mathrm{CCl}$ index concludes $\mathrm{CCl}$ index and age. ADO index concludes age, pulmonary function (FEVI\%) and dyspnea score (mMRC score). COTE index concludes 12 comorbidities (Solid organ tumors, Anxiety, Cirrhosis, Atrial Fibrillation, Atrial flutter, Diabetes with Neuropathy, Pulmonary Fibrosis, Congestive Heart Failure, Gastroduodenal Ulcer, Coronary Artery Disease). CODEx index concludes age adjusted CCl, airflow obstruction (FEVI\%), dyspnea score (mMRC score), history of severe exacerbation (hospitalization history). BODEx index was composed of BMI, airflow obstruction (FEVI\%), dyspnea score (mMRC score) and history of severe exacerbation (hospitalization history). (B) Nomogram plot describe the composition of new score to predict exacerbation in patients with COPD by calculating each individuals' exacerbation risk. First, we locate the range of each variable on the horizontal scale, and draw a line vertically to the bottom scores line to determine the corresponding points. Then we sum up the points of all of the five variables and locate the total score on the total score line. Finally, we draw a vertical line from the dot on the total score line to three upward risk probability to predict the risk of exacerbation. (C) ROC analysis for the new score to predict acute exacerbation in population of cluster I and cluster 3 . 

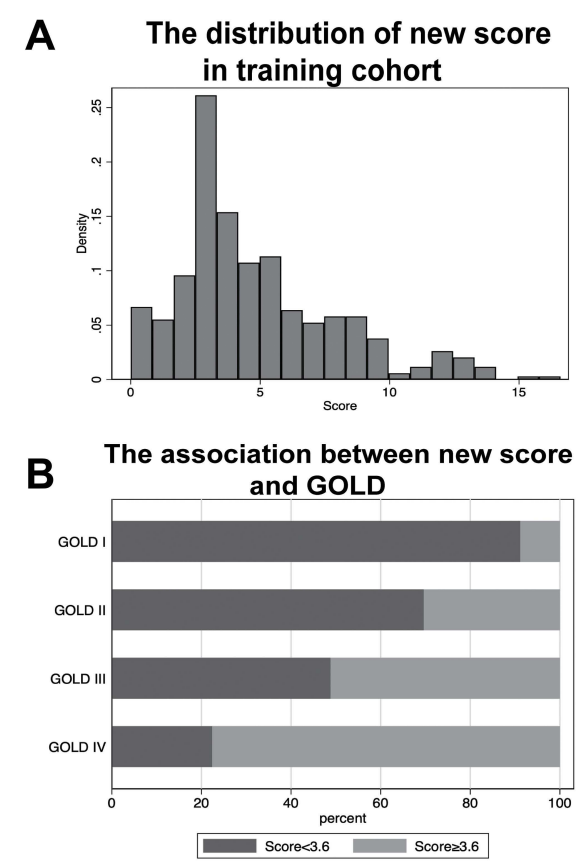

C The association between new score and $\mathrm{MMRC}$ score

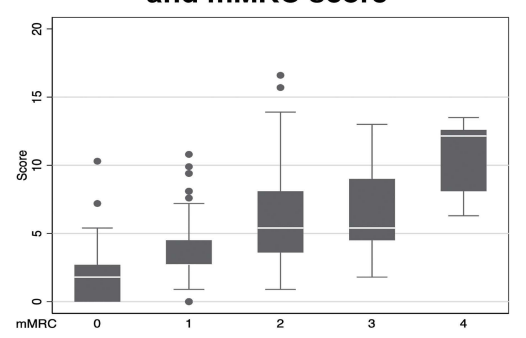

D Linear regression between FEV1 and new score

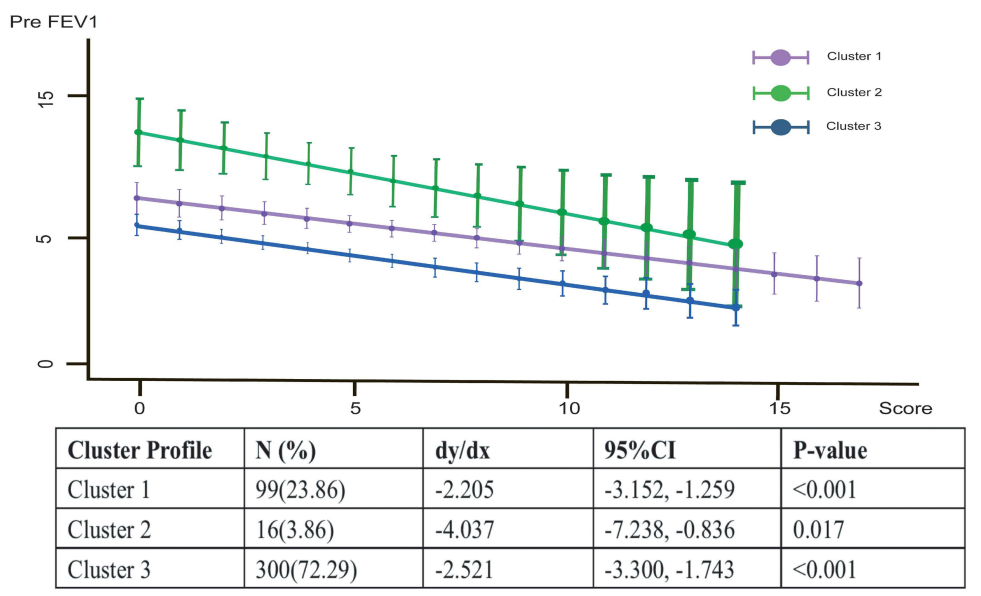

E Linear regression between FEV1/FVC and new score Pre FEV1/FVC

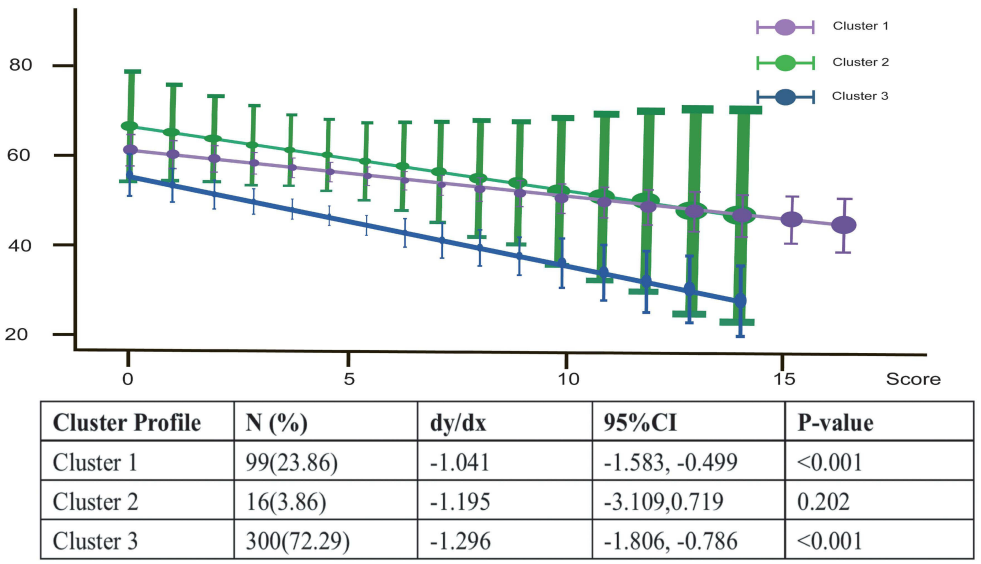

Figure 5 The distribution of new score in training cohort. (A) The distribution of new score among COPD patients derived from training cohort. (B) Horizontal bar chart shows the fraction of high and low score classified by GOLD and cut-off value of 3.6 is determined by sensitivity and specificity in the ROC. (C) The new score is compared among patients with different degree of mMRC score. (D and E) The correlation is assessed by Spearman linear regression between lung function test (FEVI\% (D) and FEVI/ FVC (E)) and score in three different clusters.

study showed that angina was one of the independent contributors to exacerbation after coordinating confounding factors in COPD patients. The tight relationship between IHD and COPD is beyond a shared risk factor. $^{19,20}$ There's more and more evidence supporting that they may share the downstream mechanistic pathway, including accelerated senescence, inflammation response and oxidative stress. ${ }^{21,22}$ With regard to the cardiovascular disease, we also explored the myocardial infarction, heart failure, angina, arrhythmias, peripheral vascular disease and hypertension. Among them, hypertension and angina present strong association with acute exacerbation of COPD. Maaike Giezeman found that heart disease contributed to lower health status and worse symptom in COPD. ${ }^{23}$ Yilmaz Gunes proposed that stable angina lacked noninvasive diagnostic methods to follow-up compared with full use of clinical diagnosis in unstable angina. ${ }^{24}$ A good prediction score may fill the gap in the future. Thibaud Soumagne's results even reinforced the application of cardiovascular risk factor score in mild-moderate

Table 4 Relationship Between Exacerbation and Score in Validation Cohort

\begin{tabular}{|l|l|l|l|l|}
\hline & OR & AUC & $\mathbf{9 5 \%} \mathbf{C I}$ & $\boldsymbol{P}$-value \\
\hline Total Exacerbation & 1.258 & 0.720 & $1.105-1.432$ & 0.001 \\
\hline Mild Exacerbation & 1.307 & 0.736 & $1.136-1.503$ & $<0.001$ \\
\hline Moderate Exacerbation & 0.829 & 0.441 & $0.352-1.954$ & 0.669 \\
\hline Severe Exacerbation & 0.921 & 0.449 & $0.643-1.320$ & 0.655 \\
\hline
\end{tabular}


COPD. ${ }^{25}$ Organ et al postulated that cardiac comorbidity in COPD patients might play a vital role in the severity of disease especially exacerbation which was corresponding to our conclusion. ${ }^{26}$ As we known, COPD also influences IHD at some rate and even exerts impact on postmyocardial infarction outcomes. ${ }^{27}$

Many researchers suggested a link between lung cancer and COPD. ${ }^{28-30}$ They shared common risk factors - cigarette smoke and genetic susceptibility. COPD patients suffered from a higher risk of lung cancer. Interestingly, COPD associated lung cancer presented distinct clinical features. For example, their lesions are prone to become positioned in the center, more invasive, shorter volume compared with lung cancer alone. ${ }^{31}$ Jae II Shin proposed that cigarette smoke resulted in the low expression of miRNA let-7, and the latter could mediate airway immunity in COPD and at the same time suppress proliferation of tumor in lung cancer. ${ }^{32}$ However, there's no significant evidence proving that COPD patients exacerbate more frequently when co-existing lung cancer in our study. It may be due to the population we targeted. With the widespread application of high-resolution CT scan (HRCT) testing in Shanghai and extensive health education for lung nodules, many patients were excluded via early detection and early treatment in surgery system as first consultation department. That is the reason why we found a lower incidence rate of lung cancer in COPD patients than we expected. The incidence rate of other comorbidities we explored was also comparatively low. It's necessary for us to seek more kinds and underlying chronic conditions in the subsequent study.

Allergic diseases like allergic rhinitis, allergic dermatitis and asthma were all explored in our study. Combined allergic rhinitis and asthma syndrome is a new concept which means they share the same characteristics like the same etiological agents, inflammatory cell profiles and therapeutic treatment. ${ }^{33}$ However, we surprisingly found that allergic rhinitis is a protective risk factor while asthma is a risk factor for COPD exacerbation. Our result was also consistent with Joo Kyung Kim's study, previous history of pneumonia rather than allergic rhinitis is the risk factor for COPD exacerbation. ${ }^{34}$ Allergic rhinitis and asthma are likely to be two diseases of the same airway. Th2-driven inflammation response and Th2 cytokines (IL-4, IL-13) are established to be critical in both of them. ${ }^{33}$ It is widely believed that allergic inflammation response exacerbates the illness. Daniel B. Jamieson's research revealed that in individuals with COPD, allergic phenotype was more likely to have respiratory symptoms. ${ }^{35}$ On the contrary, anti-Th2 response shows great efficacy in reducing the incidence of acute exacerbation. Although allergic rhinitis and asthma are in tight correlation, they are not exactly the same, and allergic rhinitis still has its uniqueness. ${ }^{36} \mathrm{We}$ found that allergic rhinitis is usually the predictor of asthma at early stage in the guideline. ${ }^{37}$ Nowadays COPD is characterized as the imbalance of Th1/Th2 which is positively correlated with the severity of disease. $^{38,39}$ Therefore, we postulate that the mild allergic status gives some protection through slowing the process of Th1/Th2 imbalance. When developing asthma, especially co-existing with COPD, it always indicates the disorder of immune status. Until now, the role of allergic rhinitis in COPD exacerbation is still a controversial problem. Cellular mechanism and immune balance need further intensive investigation.

Although the exacerbation event of COPD is inextricably linked to the prognosis, the problem has not yet been resolved. While in fact, these comprehensive score systems (CCI, ageadjusted CCI, COTE, BODEx, ADO) incorporate similar components like age, BMI, dyspnea score (mMRC), comorbidity, degree of airflow limitation (FEV1\%). Of them, CCI index is widely used as a quantifiable indicator of comorbidities. It is a conventional score system composed of 16 diseases which is used to evaluate the severity and prognosis of COPD. A variety of parameters in CCI make it have a lower clinical operation. We all known measurable parameters in COPD are limited but the configuration of these parameters is infinite. So what we can do is to take full advantage of these profiles and develop the optimal combination.

Apart from comorbidity, exacerbation history is one of the most important indicators to predict COPD exacerbation. The systematic index CODEx incorporates the variable severe exacerbation history. Some studies provided the evidence to support that both CODEx and BODEx are useful for predicting hospitalization and exacerbation in outpatients with advanced COPD. ${ }^{11,40,41}$ In the subsequent study, we will pay more attention to collect the information related to exacerbation history. And Cbiung-Zuei Chen et al found more superior predictive ability of BODEx than GOLD classification for mortality in elder COPD individuals. ${ }^{42}$ However, the BODEx indices are comorbidity non-specific indices. From a special perspective, we combined these conventional indices with comorbidity-related parameters to develop a new risk score. After combining with comorbidity profiles, its predictive capacity is significantly elevated. 
There are, however, several drawbacks to be considered. First, after cluster analysis, we found that patients co-exiting with cardiovascular and neoplastic disease in cluster 2 are far fewer than other clusters, so further larger sample size studies are needed. Secondly, we did not collect data about the exact date of exacerbation event and thus we cannot estimate the 30-day exacerbation or hospitalization. Thirdly, more attention will be paid to the information collection including the kinds of comorbidities, underlying chronic conditions and exacerbation history in subsequent study. Although the sample size in our cohort was limited now, further validation of external large-sample population is still required to validate the stability of the novel risk score.

\section{Conclusions}

In our study, 3 distinct sub-phenotypes were identified using cluster analysis of comorbidity profiles in the population of stable COPD. Importantly, the novel risk score we developed for assessing the risk of acute exacerbation showed good internal consistency among the population in our study. The risk score performed well in identifying individuals at high risk of exacerbation among specified sub-phenotype (Cluster 1). To some extent, we hope our results may provide some assistance for assessing the risk of acute exacerbation of COPD.

\section{Data Sharing Statement}

The datasets during and/or analyzed during the current study available from the corresponding author on reasonable request.

\section{Ethical Approval and Consent to Participate}

The study was approved by Ethic Committee of Huadong Hospital and the approved number of ethic committee is 20180064. The study adhered to the Declaration of Helsinki. All written informed consents were available.

\section{Author Contributions}

All authors contributed to data analysis, drafting or revising the article, gave final approval of the version to be published, and agree to be accountable for all aspects of the work.

\section{Funding}

This work was supported by the National Natural Science Foundation of China (81871100, 81600056),National key
R\&D Programe of China(2020YFC2009001), Shanghai Natural Science Foundation (18ZR1412900), Bethune Research and Development Fund Project (BJRW2020002J) and Guanghua Medical Fund Project.

\section{Disclosure}

The authors declare that they have no competing interests.

\section{References}

1. Miravitlles M, Ribera A. Understanding the impact of symptoms on the burden of COPD. Respir Res. 2017;18(1):67. doi:10.1186/ s12931-017-0548-3

2. Lopez-Campos JL, Tan W, Soriano JB. Global burden of COPD Respirology. 2016;21(1):14-23. doi:10.1111/resp.12660

3. Zhu B, Wang Y, Ming J, Chen W, Zhang L. Disease burden of COPD in China: a systematic review. Int J Chron Obstruct Pulmon Dis. 2018;13:1353-1364. doi:10.2147/COPD.S161555

4. Negewo NA, Gibson PG, McDonald VM. COPD and its comorbidities: impact, measurement and mechanisms. Respirology. 2015;20 (8):1160-1171.

5. Smith MC, Wrobel JP. Epidemiology and clinical impact of major comorbidities in patients with COPD. Int J Chron Obstruct Pulmon Dis. 2014;9:871-888. doi:10.2147/COPD.S49621

6. Maselli DJ, Bhatt SP, Anzueto A, et al. Clinical epidemiology of COPD: insights from 10 years of the COPDGene Study. Chest. 2019;156(2):228-238. doi:10.1016/j.chest.2019.04.135

7. Mantero M, Rogliani P, Di Pasquale M, et al. Acute exacerbations of COPD: risk factors for failure and relapse. Int $J$ Chron Obstruct Pulmon Dis. 2017;12:2687-2693. doi:10.2147/COPD.S145253

8. Halpin DM, Miravitlles M, Metzdorf N, Celli B. Impact and prevention of severe exacerbations of COPD: a review of the evidence. Int J Chron Obstruct Pulmon Dis. 2017;12:2891-2908. doi:10.2147/ COPD.S139470

9. Maters GA, de Voogd JN, Sanderman R, Wempe JB. Predictors of all-cause mortality in patients with stable COPD: medical co-morbid conditions or high depressive symptoms. COPD. 2014;11 (4):468-474. doi:10.3109/15412555.2014.898026

10. Global initiative for chronic global initiative for chronic obstructive lung disease. Available from: http://gold-copd.html. Accessed June 28, 2021.

11. Almagro P, Martinez-Camblor P, Miravitlles M, et al. External validation and recalculation of the CODEX index in COPD patients. A 3CIAplus Cohort Study. COPD. 2019;16(1):8-17. doi:10.1080/ 15412555.2018.1484440

12. Divo M, Cote C, de Torres JP, et al. Comorbidities and risk of mortality in patients with chronic obstructive pulmonary disease. Am J Respir Crit Care Med. 2012;186(2):155-161. doi:10.1164/ rccm.201201-0034OC

13. Li Y, Ragland M, Austin E, et al. Co-morbidity patterns identified using latent class analysis of medications predict all-cause mortality independent of other known risk factors: the COPDGene $((\mathrm{R}))$ Study. Clin Epidemiol. 2020;12:1171-1181. doi:10.2147/CLEP. S279075

14. Iheanacho I, Zhang S, King D, Rizzo M, Ismaila AS. Economic burden of Chronic Obstructive Pulmonary Disease (COPD): a systematic literature review. Int J Chron Obstruct Pulmon Dis. 2020;15:439-460. doi:10.2147/COPD.S234942

15. Ouaalaya EH, Falque L, Dupis JM, et al. Susceptibility to frequent exacerbation in COPD patients: impact of the exacerbations history, vaccinations and comorbidities? Respir Med. 2020;169:106018. doi:10.1016/j.rmed.2020.106018 
16. Vanfleteren L, Fabbri LM. Self-management interventions in COPD patients with multimorbidity. Eur Respir J. 2019;54(5):1901850. doi:10.1183/13993003.01850-2019

17. Maricoto T, Santos D, Carvalho C, Teles I, Correia-de-sousa J, Taborda-Barata L. Assessment of poor inhaler technique in older patients with asthma or COPD: a predictive tool for clinical risk and inhaler performance. Drugs Aging. 2020;37(8):605-616. doi:10.1007/s40266-020-00779-6

18. Kunisaki KM, Dransfield MT, Anderson JA, et al. Exacerbations of chronic obstructive pulmonary disease and cardiac events. A post hoc cohort analysis from the SUMMIT randomized clinical trial. Am J Respir Crit Care Med. 2018;198(1):51-57. doi:10.1164/rccm.201711-2239OC

19. Campo G, Pavasini R, Biscaglia S, Contoli M, Ceconi C. Overview of the pharmacological challenges facing physicians in the management of patients with concomitant cardiovascular disease and chronic obstructive pulmonary disease. Eur Heart $J$ Cardiovasc Pharmacother. 2015;1(3):205-211. doi:10.1093/ehjcvp/pvv019

20. Roversi S, Fabbri LM. Lung and heart interaction: chronic obstructive pulmonary disease and ischemic heart disease. G Ital Cardiol. 2018;19(3):153-160. doi:10.1714/2883.29073

21. Campo G, Pavasini R, Malagu M, et al. Chronic obstructive pulmonary disease and ischemic heart disease comorbidity: overview of mechanisms and clinical management. Cardiovasc Drugs Ther. 2015;29(2):147-157. doi:10.1007/s10557-014-6569-y

22. Carter P, Lagan J, Fortune C, et al. Association of cardiovascular disease with respiratory disease. J Am Coll Cardiol. 2019;73 (17):2166-2177. doi:10.1016/j.jacc.2018.11.063

23. Giezeman M, Hasselgren M, Lisspers K, et al. Influence of comorbid heart disease on dyspnea and health status in patients with COPD a cohort study. Int $J$ Chron Obstruct Pulmon Dis. 2018;13:3857-3865. doi:10.2147/COPD.S175641

24. Gunes Y, Tuncer M, Guntekin U, et al. Reliability of symptoms suggestive of angina in patients with chronic obstructive pulmonary disease. Arq Bras Cardiol. 2009;92(5):334-338, 351-335, 364-338. doi:10.1590/s0066-782x2009000500005

25. Soumagne T, Guillien A, Roche N, et al. In patients with mild-tomoderate COPD, tobacco smoking, and not COPD, is associated with a higher risk of cardiovascular comorbidity. Int J Chron Obstruct Pulmon Dis. 2020;15:1545-1555.

26. Ogan N, Gunay E, Ekici B, et al. Morphological overview of cardiovascular comorbidities in chronic obstructive pulmonary disease: frank's sign. Heart Lung. 2020;49(3):331-335. doi:10.1016/j.hrtlng.2020.01.008

27. Salisbury AC, Reid KJ, Spertus JA. Impact of chronic obstructive pulmonary disease on post-myocardial infarction outcomes. Am J Cardiol. 2007;99(5):636-641. doi:10.1016/j.amjcard.2006.09.112

28. Mouronte-Roibas C, Leiro-Fernandez V, Fernandez-Villar A, BotanaRial M, Ramos-Hernandez C, Ruano-Ravina A. COPD, emphysema and the onset of lung cancer. A systematic review. Cancer Lett. 2016;382(2):240-244. doi:10.1016/j.canlet.2016.09.002

29. Eapen MS, Hansbro PM, Larsson-Callerfelt AK, et al. Chronic obstructive pulmonary disease and lung cancer: underlying pathophysiology and new therapeutic modalities. Drugs. 2018;78 (16): 1717-1740

Journal of Inflammation Research

\section{Publish your work in this journal}

The Journal of Inflammation Research is an international, peerreviewed open-access journal that welcomes laboratory and clinical findings on the molecular basis, cell biology and pharmacology of inflammation including original research, reviews, symposium reports, hypothesis formation and commentaries on: acute/chronic inflammation; mediators of inflammation; cellular processes; molecular

Submit your manuscript here: https://www.dovepress.com/journal-of-inflammation-research-journal
30. Sandelin M, Mindus S, Thuresson M, et al. Factors associated with lung cancer in COPD patients. Int J Chron Obstruct Pulmon Dis. 2018;13:1833-1839. doi:10.2147/COPD.S162484

31. Dai J, Yang P, Cox A, Jiang G. Lung cancer and chronic obstructive pulmonary disease: from a clinical perspective. Oncotarget. 2017;8 (11):18513-18524. doi:10.18632/oncotarget.14505

32. Shin JI, Brusselle GG. Mechanistic links between COPD and lung cancer: a role of microRNA let-7? Nat Rev Cancer. 2013;14(1):70. doi:10.1038/nrc3477-c1

33. Paiva Ferreira LKD, Paiva Ferreira LAM, Monteiro TM, Bezerra GC, Bernardo LR, Piuvezam MR. Combined allergic rhinitis and asthma syndrome (CARAS). Int Immunopharmacol. 2019;74:105718. doi:10.1016/j.intimp.2019.105718

34. Kim JK, Lee SH, Lee BH, et al. Factors associated with exacerbation in mild- to-moderate COPD patients. Int J Chron Obstruct Pulmon Dis. 2016;11:1327-1333. doi:10.2147/COPD.S105583

35. Jamieson DB, Matsui EC, Belli A, et al. Effects of allergic phenotype on respiratory symptoms and exacerbations in patients with chronic obstructive pulmonary disease. Am J Respir Crit Care Med. 2013;188 (2):187-192. doi:10.1164/rccm.201211-2103OC

36. Greiner AN, Hellings PW, Rotiroti G, Scadding GK. Allergic rhinitis. Lancet. 2011;378(9809):2112-2122. doi:10.1016/S0140-6736(11) 60130-X

37. Yorgancioglu A, Ozdemir C, Kalayci O, et al. [ARIA (Allergic Rhinitis and its Impact on Asthma) achievements in 10 years and future needs]. Tuberk Toraks. 2012;60(1):92-97. Indonesian. doi: $10.5578 / \mathrm{tt} .3734$

38. Wei B, Sheng LC. Changes in Th1/Th2-producing cytokines during acute exacerbation chronic obstructive pulmonary disease. $J$ Int Med Res. 2018;46(9):3890-3902. doi:10.1177/0300060518781642

39. Jiang M, Liu H, Li Z, et al. ILC2s induce adaptive Th2-type immunity in acute exacerbation of chronic obstructive pulmonary disease. Mediators Inflamm. 2019;2019:3140183. doi:10.1155/2019/3140183

40. Navarro A, Costa R, Rodriguez-Carballeira M, et al. Prognostic assessment of mortality and hospitalizations of outpatients with advanced chronic obstructive pulmonary disease. Usefulness of the CODEX index. Rev Clin Esp. 2015;215(8):431-438. doi:10.1016/j. rce.2015.06.004

41. Hadda V, Madan K, Mohan A. CODEX index and prognosis of patients with exacerbation of COPD. Chest. 2014;145(5):1172. doi:10.1378/chest.13-2516

42. Chen $\mathrm{CZ}$, Ou CY, Yu CH, Yang SC, Chang HY, Hsiue TR. Comparison of global initiative for chronic obstructive pulmonary disease 2013 classification and body mass index, airflow obstruction, dyspnea, and exacerbations index in predicting mortality and exacerbations in elderly adults with chronic obstructive pulmonary disease. J Am Geriatr Soc. 2015;63(2):244-250. doi:10.1111/jgs.13258

mechanisms; pharmacology and novel anti-inflammatory drugs; clinical conditions involving inflammation. The manuscript managemen system is completely online and includes a very quick and fair peerreview system. Visit http://www.dovepress.com/testimonials.php to read real quotes from published authors. 